\title{
Cuidados para optimizar la atención en recién nacidos con malformaciones congénitas
}

Care to optimize the care of newborns with congenital malformations

\author{
Adisnay Rodríguez-Plasencia \\ ua.adisnayrodriguez@uniandes.edu.ec \\ Universidad Regional Autónoma de los Andes, Ambato \\ Ecuador \\ https://orcid.org/0000-0002-5293-2817 \\ Juana Priscila Moya-Arcos \\ ea.juanapma66@uniandes.edu.ec \\ Universidad Regional Autónoma de los Andes, Ambato \\ Ecuador \\ https://orcid.org/0000-0003-3546-3948 \\ Ligia Maribel Sánchez-Morales \\ ea.ligiamsm92@uniandes.edu.ec \\ Universidad Regional Autónoma de los Andes, Ambato \\ Ecuador \\ https://orcid.org/0000-0001-9326-9276
}

Recepción: 10 de agosto 2021

Revisado: 15 de septiembre 2021

Aprobación: 15 de noviembre 2021

Publicación: 01 de diciembre 2021 
Revista Arbitrada Interdisciplinaria de Ciencias de la Salud. SALUD Y VIDA

Volumen 5. Número 1. Año 5. Especial. 2021

Hecho el depósito de Ley: FA2016000010

ISSN: $2610-8038$

FUNDACIÓN KOINONIA (F.K).

Santa Ana de Coro, Venezuela.

Adisnay Rodríguez Plasencia; Juana Priscila Moya Arcos; Ligia Maribel Sánchez Morales

\section{Sra. Editora:}

Se presenta un caso clínico, donde Madre refiere que aproximadamente hace 8 horas presenta dolor abdominal tipo contracción en hipogastrio, irradiado a región lumbar de moderada intensidad por lo que acude a centro de Salud donde es atendido mediante un parto eutócico sin complicaciones, se recibe un RN a las 19:30pm de sexo masculino vivo, llanto fuerte, clampeo oportuno inmediato cordón umbilical y pasa alojamiento conjunto, con peso de $2530 \mathrm{~kg}$, Talla: $47 \mathrm{~cm}$, PC: $33 \mathrm{~cm}$, APGAR: 9/9 Al examen físico $\mathrm{RN}$, identifican labio leporino y paladar hendido, por lo que es referido a esta casa de salud.

\section{Examen físico}

Recién nacido vivo activo y reactivo al manejo, piel ictérica zona Kramer IV Cabeza: Fontanela anterior normotensa. Ojos: Normoreactivos Icónicos. Nariz: Fosas Nasales permeables. Oídos: De implantación normal. Boca: Presencia de falta de continuidad del labio superior lado derecho "labio leporino" y ausencia de paladar. Cuello: Central, móvil, no se palpa adenopatías. Pulmones: A la inspección buena entrada y salida de aire. Cardiopulmonar: Ruidos cardiacos: rítmicos no se auscultan soplos. Abdomen: Suave depresible, RHA presentes no distendido. Extremidades: Tono, Fuerza y movilidad conservada. ENE: Reflejo de moro completo, reflejo de búsqueda y succión presente.

\section{Diagnóstico}

Malformación congénita del paladar, Labio Leporino. CIE 369. (1) (2) (3) (4) (5) (6).

\section{Planes de tratamiento médico}

Ingreso a Neonatología, cuna corriente, colocar Sonda orogástrica (SOG), administrar Leche Materna, balance hídrico, vigilar tolerancia Oral, Vigilar signos de Ictericia, Tramitar obturador de techo palatino, Interconsulta con Psicología, Interconsulta con Cirugía 
Revista Arbitrada Interdisciplinaria de Ciencias de la Salud. SALUD Y VIDA

Volumen 5. Número 1. Año 5. Especial. 2021

Hecho el depósito de Ley: FA2016000010

ISSN: $2610-8038$

FUNDACIÓN KOINONIA (F.K).

Santa Ana de Coro, Venezuela.

Adisnay Rodríguez Plasencia; Juana Priscila Moya Arcos; Ligia Maribel Sánchez Morales

Plástica en el Hospital Baca Ortiz, Interconsulta con Genetista, pendiente realizar Ecografía trasfontanelar, Pendiente vacuna BCG.

\section{Plan de cuidados de enfermería}

Cuna corriente más abrigo para mantener temperatura de 36.5 y $37.5^{\circ} \mathrm{C}$ Cabecera elevada a $30 \stackrel{\circ}{\circ}$, Control de Signos Vitales cada tres horas, Control de diuresis Horaria, Peso Diario, Administrar leche materna $20 \mathrm{cc}$ c/3h por SOG, vigilar tolerancia oral, vigilar signos de ictericia, higiene de placa palatina $8 \mathrm{am}, 12 \mathrm{pm}, 20 \mathrm{pm}$ con agua cepillo de dientes y jabón líquido, colocación de placa previa utilización de crema adhesiva corega ultra, apoyo en manejo materno, educación a la madre.

Como estrategia, se elaboró una guía de cuidados dirigida a las madres para optimizar la atención en los recién nacidos con malformación congénita del Hospital General Docente Ambato.

Se fundamentaron los conceptos teóricos necesarios para el desarrollo de un estudio sobre recién nacidos con malformación congénita, obteniendo los conocimientos necesarios sobre los cuidados especiales, que se deben tener en estos pacientes.

Se logró Identificar el nivel de conocimiento de la madre sobre malformaciones congénitas en el recién nacido mediante la aplicación de un cuestionario, donde se obtuvo que las madres, desconocen sobre el concepto, las causas, factores de riesgo, prevención y la posición adecuada de alimentar a su bebe que padece de este tipo de patología.

Se definieron los aspectos de una estrategia de enfermería para el manejo y atención de recién nacidos que presentan malformación congénita para evitar complicaciones inmediatas como broncoaspiración, desnutrición, mediatas tales como alteraciones auditivas, Infecciones o hipoacusia y siendo las tardías, alteraciones, retardo o deficiencia de lenguaje y presentando problemas odontológicos. 
Revista Arbitrada Interdisciplinaria de Ciencias de la Salud. SALUD Y VIDA

Volumen 5. Número 1. Año 5. Especial. 2021

Hecho el depósito de Ley: FA2016000010

ISSN: $2610-8038$

FUNDACIÓN KOINONIA (F.K).

Santa Ana de Coro, Venezuela.

Adisnay Rodríguez Plasencia; Juana Priscila Moya Arcos; Ligia Maribel Sánchez Morales

Recomendamos la elaboración de más estudios de casos sobre malformación congénitas en los recién nacidos y controles con una población amplia y a gran escala para conocer la verdadera realidad de nuestra localidad e implementar estrategias de prevención adecuadas mediante la educación de las madres en centros de salud.

\section{CONFLICTO DE INTERÉS}

Los autores declaran que no tienen conflicto de interés en la publicación del artículo.

\section{FINANCIAMIENTO}

No monetario.

\section{AGRADECIMIENTO}

A la Universidad Regional Autónoma de los Andes, por apoyar el desarrollo de la investigación.

\section{REFERENCIAS}

1. Worley ML, Patel KG, Kilpatrick LA. Cleft Lip and Palate. Clin Perinatol. 2018;45(4):661-678. doi:10.1016/j.clp.2018.07.006

2. Díaz Casado GH, Díaz Grávalos GJ. Defectos de cierre orofaciales: paladar hendido y labio leporino. Una revisión bibliográfica [Orofacial closure defects: cleft lip and palate. A literature review]. Semergen. 2013;39(5):267-271. doi:10.1016/j.semerg.2012.08.003

3. Kawalec A, Nelke K, Pawlas K, Gerber H. Risk factors involved in orofacial cleft predisposition - review. Open Med (Wars). 2015;10(1):163-175. Published 2015 Feb 5. doi:10.1515/med-2015-0027 
4. Pereira AV, Fradinho N, Carmo S, et al. Associated Malformations in Children with Orofacial Clefts in Portugal: A 31-Year Study. Plast Reconstr Surg Glob Open. 2018;6(2):e1635. Published 2018 Feb 9. doi:10.1097/GOX.0000000000001635

5. Maximino LP, Marcelino FC, Cavalheiro MG, et al. Auditory and language skills in children with cleft lip and palate [published online ahead of print, $2021 \mathrm{Feb} 18$ ]. Acta Otorrinolaringol Esp (Engl Ed). 2021;S0001-6519(20)30193-X. doi:10.1016/j.otorri.2020.11.002

6. Abdel-Ghaffar HS, Abdel-Aziz NGE, Mostafa MF, Osman AK, Thabet NM. Cetamina como adjuvante de bupivacaína em bloqueio do nervo infraorbitário para analgesia após correção de lábio leporino [Ketamine as an adjunct to bupivacaine in infra-orbital nerve block analgesia after cleft lip repair]. Braz $J$ Anesthesiol. 2018;68(3):266-273. doi:10.1016/j.bjan.2018.01.007 